\title{
La prédiction des marées théorisation et mécanisation AuX 19e et 20e siecles
}

Marie-José Durand-Rochard

\begin{abstract}
Résumé
Le rôle des ingénieurs dans l'histoire des mathématiques est d'autant plus souvent passée sous silence que cette histoire est en général étudiée du point de vue de l'université et des institutions académiques. Pourtant, à tenir compte des théories du langage les plus récentes, et qui intègre sa dimension pragmatique, la signification des mathématiques ne relève pas seulement de sa validité logique, mais de l'ensemble des contextes dans lesquelles elle interagit socialement. Cet article développe à la fois l'analyse de cette problématique, et une étude de cas significative, celle de la prédiction des marées depuis la théorie de Laplace.
\end{abstract}

Mots-clés: mathématiques, langage, pragmatique, étude de cas, machines mathématiques, prévision des marées

Abstract

The role of engineers in history of mathematics is more often overlooked that this history is usually studied from the view of academic institutions. However, if we take account of latest theories of language, which integrates its pragmatic dimension, the meaning of mathematics does not come only from its logical validity, but from all the contexts in which it interacts socially. This paper develops both the analysis of this problematics, and a significant case study, the prediction of tides from the theory of Laplace.

Keywords: mathematics, language, pragmatics, case study, mathematical machines, tide prediction. 
A partir du $19^{\mathrm{e}}$ siècle, le problème de la prédiction des marées convoque non seulement le physicien, l'astronome et le navigateur, mais aussi le mathématicien et l'ingénieur. A ce titre, le sujet concerne directement le thème de ces journées sur le rôle et la pratique des mathématiques dans la formation des ingénieurs. Cette convergence d'intérêts se produit alors autour de la mécanisation des instruments mathématiques, favorisée par l'industrialisation de l'Europe. Avant d'entrer dans le vif du sujet, je voudrais montrer en quoi ce type de convergence fait directement partie de l'histoire des mathématiques, pour peu que soit envisagée la question de ce qui rend les mathématiques pertinentes dans un contexte donné.

\section{Des mathématiques signifiantes}

Au collège, au lycée comme au niveau du supérieur, les enseignants sont régulièrement soumis à une question récurrente chez les élèves et les étudiants: "à quoi ça sert, les mathématiques ? ", une question qui signifie bien plutôt : "à quoi ça me sert d'apprendre des mathématiques? ". En somme, bien que les mathématiques soient partout présentes dans les outils technologiques qu'ils manipulent ou voient fonctionner au quotidien - ordinateur, téléphone portable, four à micro-ondes -, les élèves ne sont sensibles qu'à ces effets pratiques des mathématiques, et n'aperçoivent ni où ni comment elles interviennent effectivement.

La réponse à une telle question n'est pas évidente pour les enseignants auxquels elle s'adresse. La plupart du temps, ils n'en savent eux-mêmes pas grand-chose, puisqu'ils ont reçu en général un enseignement purement théorique, où les implications sociétales ne sont que rarement évoquées, et laissées à la libre initiative des formateurs. La situation présente évolue quelque peu par rapport à cet état de fait, mais l'évolution est lente, et souvent limitée à une histoire conceptuelle qui reste la plupart du temps très internaliste. Dans leur propre formation, les enseignants ont plutôt été bercés par le discours selon lequel la vérité des mathématiques ne vaut que pour elle- même, et qu'elle est sans rapport avec la réalité du monde.

L'opposition est donc manifeste entre ce " désir de sens » de la part des élèves - souci bien légitime pour ces citoyens en herbe -, et, sinon un refus de sens de la part des enseignants, du moins la négation de l'importance du sens à donner aux mathématiques en dehors d'une structuration logique appréhendée comme intrinsèque. II est bien sûr possible d'envisager l'enseignement autrement, de vouloir apporter autre chose que des contenus strictement mathématiques, de tenter de montrer aux élèves que la rationalité se construit, qu'elle se construit en se confrontant aussi à l'irrationnel. Il n'empêche : montrer les effets d'invention prend du temps, et une telle démarche est de fait vouée à la marginalité dès lors qu'elle n'est pas intégrée dans une recomposition des programmes d'enseignement. 


\subsection{Historicité des relations entre mathématiques et langage: le souci de perfection}

Pourtant, dès que les mathématiques se trouvent abordées du point de vue de leur histoire, il apparaît qu'elles sont en permanence confrontées à bien d'autres facteurs qu'au seul souci de vérité interne. Outre le fait que la recherche mathématique avance en procédant tout autant par certitudes que par conjectures, elle est également confrontée à une multitude d'enjeux relationnels et sociétaux qui ne font pas qu'orienter les directions de recherche : elles marquent aussi le type de structuration démonstrative exigée dans un contexte spécifique ${ }^{1}$. Dès que les mathématiques sont envisagées du point de vue de leur histoire, se trouvent d'emblée réintroduits le sujet, le temps et la société, trois facteurs déterminants que l'enseignement s'évertue pourtant à éliminer radicalement des programmes.

En France, cette coupure radicale entre mathématiques et signification est souvent rapportée à l'impact du programme bourbakiste des années 1950. Elle est pourtant beaucoup plus ancienne, puisque cette coupure se trouve déjà affirmée chez Descartes qui énonce, dès 1637 , les modalités de la résolution algébrique d'un problème, sous une forme tout à fait proche de celle qui est enseignée aujourd'hui :

«Voulant résoudre quelque problème, on doit d'abord le considérer comme déjà fait, et donner des noms à toutes les lignes qui semblent nécessaires pour le construire, aussi bien à celles qui sont inconnues qu'aux autres. Puis sans considérer aucune différence entre ces lignes connues ou inconnues, on doit parcourir la difficulté, selon l'ordre qui montre le plus naturellement de tous en quelle sorte elles dépendent mutuellement les unes des autres, jusques à ce qu'on ait trouvé moyen d'exprimer une même quantité en deux façons : ce qui se nomme une Equation, car les termes de l'une de ces deux façons sont égaux à ceux de l'autre. Et on doit trouver autant de telles équations qu'on a supposé de lignes qui étaient inconnue $»^{2}$.

${ }^{1}$ C'est ainsi qu'en Chine, avant les contacts avec l'Europe, ce qui fait preuve en mathématiques relève de procédures algorithmiques, qui ont tardé à être reconnues comme démonstratives par les historiens occidentaux. Chemla \& Shuchun, Les neuf chapitres; Volkov, Alexeï, " Raisonnement par analogie dans les mathématiques chinoises ».

${ }^{2}$ Descartes, La Géométrie, p. 6. 
La résolution algébrique d'un problème s'effectue ainsi indépendamment de sa signification. Dans la même veine, au début du $19^{\mathrm{e}}$ siècle, l'algébriste anglais Charles Babbage (1791-1871) réalise les plans de sa "machine analytique »- assimilable à une calculatrice automatique à programme externe ", en se référant à l'algèbre comme à un langage des signes, un " langage du raisonnement symbolique » détenteur de sa propre autonomie, et dont il va transférer les opérations à un appareillage mécanique :

"La cause qui a surtout contribué à adapter ainsi le langage des signes peut être trouvée dans cette circonstance qu'étant elle-même une méthode de raisonnement d'une extrême généralité, l'algèbre a été découverte au moyen de l'une de ses applications particulières, celle qui concerne les nombres ...

L'objet que je propose de tenter est de séparer entièrement l'analyse ou le langage des signes de toutes ses diverses applications, en en rejetant non seulement les considérations géométriques mais aussi celles de nombre, et de montrer que, quand on l'examine de cette façon, (cette analyse) se résout ellemême en propositions qui sont purement identiques, ou au moins que la signification de toute équation ne correspond à rien d'autre qu'à écrire que, lorsque toutes les opérations indiquées de chaque côté sont effectuées, toute lettre qui intervient d'un côté interviendra aussi de l'autre côté, précisément dans les mêmes circonstances $»^{1}$.

Sur ses traces, et donc sensiblement à la même époque, lorsque le logicien George Boole (1805-1864) ose le premier rapprochement entre logique et mathématiques, ou plutôt entre logique et algèbre, il le fait au nom de cette même conception symbolique d'une algèbre dont les processus opératoires sont coupés de toute référence à leur signification :

"Les conditions d'un raisonnement valide, au moyen des symboles, sont les suivantes:

1. qu'une interprétation fixée est assignée aux symboles utilisés dans l'expression des données, et que les lois de combinaison de ces symboles sont correctement déterminées à partir de cette interprétation,

2. que les processus formels de solution ou de démonstration soient conduits en obéissant à toutes les lois déterminées ci-dessus, sans s'occuper de la question de l'interprétation des résultats particuliers obtenus,

3. que le résultat final soit interprétable, et qu'il soit effectivement interprété, en accord avec le système d'interprétation qui a été employé dans l'expression

${ }^{1}$ Babbage, The Philosophy of Analysis, pp. 42-43. 
des données $»^{1}$.

L'idée d'une coupure entre calcul algébrique et signification des résultats est donc une revendication très ancienne, et qui intervient d'ailleurs dans des situations très spécifiques, où l'algèbre elle-même est en quête d'une identité propre. Descartes participe à un processus d'unification entre différentes amorces de symbolisation de l'algèbre élaborées localement. Au $17^{\mathrm{e}}$ siècle, la partie n'est pas gagnée pour imposer l'algèbre comme nouvelle méthode, face à la géométrie et l'arithmétique, qui disposent alors de méthodologies reconnues, et historiquement enracinées dans l'édifice théorique des Eléments d'Euclide. Elle ne l'est toujours pas au début du $19^{\mathrm{e}}$ siècle à Cambridge, lorsqu'au sein de tout un réseau d'algébristes, Babbage cherche à imposer l'algèbre comme science dans cette université anglicane, où elle est plutôt considérer comme un art utile au commerce et aux assurances, trop marquée par les processus d'invention : remplacer les nombres par des lettres ne suffit pas, encore faut-il structurer le calcul par des propriétés opératoires spécifiques, en voie d'explicitation à cette époque. Et c'est fort de cette conception du calcul que Boole substitue des règles universelles aux règles d'Aristote articulant les syllogismes concluants. C'est au prix de cette coupure radicale entre le calcul et une signification devenant « interprétation » subordonnée au calcul que l'algèbre sera reconnue comme science théorique et abstraite.

De fait, cette coupure ne s'impose pas d'elle-même. Elle s'appuie sur une conception du langage elle aussi affirmée par Descartes, selon laquelle le langage serait constitué d'une grammaire - qui en régirait la structure - et d'un dictionnaire - qui attribuerait les significations. $\mathrm{Au}$ nom de cette conception, élaborer une grammaire rigoureuse - débarrassée de ses exceptions - et un dictionnaire à entrées uniques permettrait d'obtenir une langue parfaite. Et c'est bien là la tâche du philosophe : purifier le langage, le débarrasser des "corruptions de l'usage ", pour aboutir à une adéquation parfaite de ce langage au réel. Ce rôle purificateur du philosophe mathématicien ne s'arrête pas avec la Mathesis Universalis de Descartes. Leibniz le réaffirme dans son projet de Caractéristique Universelle fondée sur le modèle de l'algèbre, et les sirènes des années 1970 assimilant cerveau et ordinateur ${ }^{2}$ le convoqueront à leur tour, supposant une rationalité logique comme perfection sous-jacente aux imperfections de l'humain. Que cette perfection soit envisagée comme à retrouver ou à construire, la perspective théologique n'est pas loin.

${ }^{1}$ Boole, Les lois de la pensée, pp. 82-83.

${ }^{2}$ L'ordinateur est alors réduit à une stricte et « pure » matérialisation de la logique, ce qui est loin d'être le cas. Ramunni, Girolamo, « L'ordinateur entre continuité et rupture ». 


\subsection{Des mathématiques au sein des échanges langagiers}

Pourquoi l'enseignement des mathématiques - voire la conception du calculpersisterait-elle aujourd'hui à s'appuyer sur une conception du langage qui date du $17^{e}$ siècle $e^{1}$ ? Pourquoi ne pourrait-il pas investir d'autres conceptions du langage aujourd'hui existantes ? Les travaux de plusieurs linguistes ouvrent la possibilité de réintégrer dans les mathématiques les différents facteurs de leur signification pleine et entière dans la société où elles sont pratiquées et transmises. Trois d'entre eux me paraissent tout à fait porteurs de cette potentialité qu'offre une approche pragmatique et diachronique en linguistique pour renouveler la conception des mathématiques en tant que discours de rationalité.

John Austin (1911-1960), initiateur de la pragmatique en linguistique ${ }^{2}$, lorsqu'il parle des " actes de langage ", insiste sur le fait que le langage n'est pas une simple description du monde, mais qu'au contraire, il est action et constitue une représentation du monde qui correspond à celle des actions de l'humain sur ce monde, représentation qui se trouve par là même susceptible à la fois de changer et de transformer le monde. Paul Ricœur (1913-2005), qui s'est longuement penché sur la question de l'interprétation ${ }^{3}$, fait intervenir non pas deux, mais trois composantes essentielles du langage, tout en montrant comment leurs rôles respectifs ont évolué historiquement les uns par rapport aux autres. Si le dictionnaire relève du registre lexical, et la grammaire du registre logique, le registre référentiel ne saurait être négligé, qui fonctionne de fait comme ontologie ${ }^{4}$. C'est dire là encore que toute expression se réfère à une certaine représentation du monde, trop souvent confondue avec sa réalité. Et une fois de plus, cette conception du monde est susceptible de changement. Dans cette perspective, l'herméneutique vaut aussi bien pour les sciences exactes que pour les autres discours théoriques. L'ouvrage de Catherine Fuchs et Bernard Victorri, La polysémie, construction dynamique du sens, traite plus directement des innombrables difficultés issues de l'approche strictement logique du langage naturel, approche que la recherche linguistique a pourtant massivement investie au moment de la naissance de l'informatique. Au contraire, pour l'équipe de linguistes, informaticiens et mathématiciens dont ces auteurs présentent le travail, la polysémie, loin d'être " corruption de l'usage » est constitutive de la richesse du langage, qu'il faut analyser comme point de départ plutôt que d'essayer de l'éliminer. Le vocabulaire est fondamentalement polysémique, et ce sont d'ailleurs les mots les plus courants qui le sont le plus. L'unicité de la signification d'un mot ou

\footnotetext{
${ }^{1}$ Je ne signifie pas ici que des conceptions plus complexes du langage n'existaient pas avant le $20^{\mathrm{e}}$
} siècle. Paul Ricœur (voir notes 7 et 8) montre en particulier ce qu'il en était de la conception aristotélicienne du langage, et les transformations qui se sont opérées au $17^{\mathrm{e}}$ siècle.

${ }^{2}$ Austin, Quand dire, c'est faire.

${ }^{3}$ Ricœur, De l'interprétation ; et Du texte à l'action, essais d'herméneutique.

${ }^{4}$ Ricœur, La métaphore vive. 
d'une expression n'est pas donnée d'emblée, elle se construit dans la confrontation des mots à l'intérieur de la phrase, et de la phrase dans son contexte. L'hypothèse de la logique classique, reprise en informatique, selon laquelle la signification d'une phrase serait la résultante des significations de ses différents éléments, se trouve ainsi battue en brèche. Cette construction du sens d'un texte se dégage ainsi d'une approche contextuelle et consensuelle qui suppose l'accord implicite entre un certain nombre d'hypothèses, qu'il est donc essentiel d'expliciter pour situer les conditions de production de la signification. L'universalité des mathématiques n'a lieu que sous certaines conditions, autour desquelles l'accord doit être établi entre les acteurs - producteurs et utilisateurs - du discours mathématique.

Comme l'écrivait déjà le philosophe John Locke (1632-1704), contemporain de Newton et fondateur de l'empirisme dans son Essay on Human Understanding, les mathématiques ne sont universelles qu'en tant qu'elles sont produites par l'esprit, et l'adéquation au réel fait intervenir tellement d'autres facteurs qu'elle relève de l'impossible pour la raison humaine ${ }^{1}$. Quoi qu'il en soit, vérité et universalité ne sauraient s'imposer d'elles-mêmes : il ne suffit pas d'avoir raison pour que tout le monde adhère à son point de vue. Les résultats de la recherche en mathématiques n'acquièrent leur caractère essentiel que pour autant qu'ils sont reconnus et transmis comme tels, et leurs implications sociétales font partie de ce qui rend les mathématiques signifiantes au moment où sont pensés les programmes d'enseignement. II est donc tout à fait urgent et raisonnable d'examiner comment peut se construire une signification communereconnue comme élément de sécurisation - entre les acteurs pour lesquels les mathématiques sont signifiantes: le chercheur, le technicien, l'enseignant, l'élève, le citoyen, l'homme et la femme de la rue.

\section{Mécanisation du calcul et prédiction des marées}

Historiquement, les mathématiques ne sont pas seulement théoriques et logiques, elles sont aussi pratiques et instrumentales. II existe des relations suivies entre instrumentation et mathématiques, qui se sont renforcées avec l'industrialisation, et qui sont tout à fait essentielles à la construction de la signification sociale accordée aux mathématiques. II est important de rappeler ici que les mathématiques n'avaient aucun caractère essentiel dans les universités au Moyen-Âge en Europe par exemple.

L'étude de la prédiction des marées offre un bon exemple de l'effectivité de ces relations entre instrumentation et mathématiques, d'autant plus caractéristiques qu'elles font émerger des

\footnotetext{
${ }^{1}$ Locke, Essai philosophique sur l'entendement humain.
} 
machines analogiques que l'histoire peine à valoriser depuis l'existence des ordinateurs, puisqu'elle tend à rattacher exclusivement ces derniers aux développements $\mathrm{du}$ calcul arithmétique digital. La prédiction des marées concerne tout autant la théorie que la pratique des mathématiques dans leur approximation du réel. Elle relève des facteurs qui vont constituer l'importance du calcul infinitésimal, et qui dépassent de beaucoup son élaboration strictement théorique telle qu'elle est présentée en cours. Lorsqu'il émerge comme discipline au $17^{\mathrm{e}}$ siècle, la manière strictement opératoire dont il est initialement conçu sous-tend des enjeux philosophiques considérables et d'évidentes limitations - dont certaines persistent aujourd'hui. Les ingénieurs du $19^{\mathrm{e}}$ siècle - dont il n'est pourtant jamais question dans l'histoire du calcul infinitésimal - vont contribuer à faire oublier les uns et à dépasser les autres en inventant les instruments-planimètres et intégraphes- qui fourniront les principes de fonctionnement de machines plus complexes: analyseurs harmoniques et analyseurs différentiels.

\subsection{Les limitations du calcul infinitésimal sur le terrain}

La présentation très formalisée sous laquelle est donnée la notion de limite dans l'enseignement, afin de fonder les concepts de dérivation et d'intégration, date en fait de la fin du $19^{\mathrm{e}}$ siècle. Elle suppose que soit constitué l'ensemble des réels comme ensemble continu de nombres, et a été élaborée par Karl Weierstrass (1825-1897), Giuseppe Peano (1858-1932) en ayant fourni ultérieurement les notations. Pour une fonction réelle $f$ par exemple, dire qu'elle a pour limite a lorsque $x$ tend vers $x_{0} s$ écrit : :

$$
\forall \varepsilon>0 \exists \alpha>0\left|\xi \angle \xi_{0}\right| \Rightarrow|\phi(\xi) \angle \alpha| \delta \varepsilon
$$

Le concept en est d'abord formulé de façon plus verbale par Augustin Louis Cauchy (17891857) dans les années 1820. Mais lorsque Isaac Newton (1642-1727) et Gottfried W. Leibniz (1646-1716) établissent le calcul infinitésimal comme discipline, ils le font sur une base beaucoup plus strictement opératoire, grâce à laquelle ils envisagent de réduire toute l'analyse mathématique à une algèbre valant aussi bien pour le fini que pour l'infini. La dérivation et de l'intégration de $x^{n}$ permettent de penser la dérivation et l'intégration terme à terme des polynômes et des séries, en tous cas pour les fonctions dites usuelles, qui sont suffisamment régulières pour être ainsi traitées. Le problème de la convergence des séries, comme condition de telles procédures sur les sommes infinies, ne se posera véritablement qu'au début du $19^{\mathrm{e}}$ siècle avec Cauchy.

Au $17^{\mathrm{e}}$ siècle, l'élaboration de Newton ne concerne pas que le calcul infinitésimal. Elle constitue les Principes mathématiques de la philosophie naturelle, titre de l'ouvrage fondamental de Newton, et base de la mathématisation de la physique à partir de tout un ensemble de concepts que le calcul infinitésimal permet d'articuler. Pour revenir aux hypothèses sous-jacentes 
qui fondent l'universalité supposée des théories scientifiques ou mathématiques, il est essentiel de souligner que la théorie newtonienne constitue la clé de voûte des travaux préalables de Copernic, Képler et Galilée, unifiant au bout d'une vingtaine de siècles les mondes sublunaire et supralunaire de la Physis aristotélicienne. Cette unification des mondes terrestre et céleste s'énonce sous forme de "lois de la nature ", ce qui était impensable jusque là. Pour Aristote, la nature est vivante, tout mouvement naturel est manifestation d'un changement porté par une pulsion de vie, aussi bien pour la nature que pour les humains - qui font partie de la nature -, et qui ne saurait être mathématisée. Cette réunification du monde, cette mathématisation des lois de la nature, ont donc un prix, un coût philosophique primordial : la nature vivante devient matière inerte. Si la science peut traiter selon les mêmes lois de tous les problèmes de mouvement, ces mouvements ne sont plus que des trajectoires parcourues dans l'espace et dans le temps, et ne concernent plus que les planètes, les corps et les machines. Une séparation nouvelle apparaît entre science et philosophie, l'une traitant de la matière, l'autre de l'humain. Au delà de l'astronomie, toutes les possibilités ouvertes à la théorie du mouvement par l'industrialisation au $19^{\mathrm{e}}$ siècle - soutenues y compris par une conception mécaniciste des corps seront sans nul doute fondamentales pour faire taire les interrogations philosophiques relevant de cette séparation, et qui ressurgissent depuis les années 1950 autour de la cybernétique et de l'informatique.

Mais quelle que soit l'ampleur de ses potentialités, le calcul infinitésimal ne peut pas tout. D'une part, il ne sait pas intégrer toutes les fonctions, même aujourd'hui. Et d'autre part, il a besoin de connaître la fonction que représente une courbe pour pouvoir calculer l'aire sous cette courbe, c'est-à-dire l'intégrale de cette fonction. Or, connaître l'aire sous une courbe, ou l'aire à l'intérieur d'une courbe fermée, est un problème très ancien, dont l'approche a d'ailleurs nourri en partie l'élaboration du calcul infinitésimal, même si, dans la pratique, la fonction correspondante est inconnue, ou n'existe pas. C'est bien la question qui se pose pour connaître l'aire d'un terrain, d'une forêt ou d'une île cartographiées, voire, à partir de certaines représentations graphiques empiriques ou théoriques, l'aire de section d'une carène de navire dans la marine, ou les volumes de terre à déplacer dans le génie civil ou militaire. Ces besoins sont typiquement de ceux qui se multiplient au $19^{\mathrm{e}}$ siècle, et pour lesquels ingénieurs, mathématiciens et artisans, puis manufacturiers, vont inventer et produire des planimètres et des intégraphes partout en Europe ${ }^{1}$. Des planimètres sont d'ailleurs toujours fabriqués aujourd'hui, notamment

${ }^{1}$ Une présentation plus détaillée du contexte de production des planimètres et des intégraphes peut être trouvée sur le site suivant: http://www.irem.univrennes1.fr/viedelirem/act anterieures/ activites_0708/ 
en Japon et aux Etats-Unis, pour être utilisés partout où ces besoins persistent sans qu'un ordinateur soit nécessairement à disposition : ainsi pour mesurer l'aire d'un banc de poisson dont l'image est obtenue par sonar, ou le débit du pétrole dans les pipe-lines.

\subsection{Les potentialités de la mécanisation du calcul infinitésimal pour l'ingénieur}

Bien que l'objet de cette présentation ne porte pas sur les planimètres, il faut pourtant en rappeler le principe et le fonctionnement, qui sont intégrés dans l'analyseur harmonique. Dans la première moitié du $19^{\mathrm{e}}$ siècle, le planimètre à cône est d'abord produit en plusieurs lieux : I'Allemagne, l'Italie, la France, l'Ecosse.

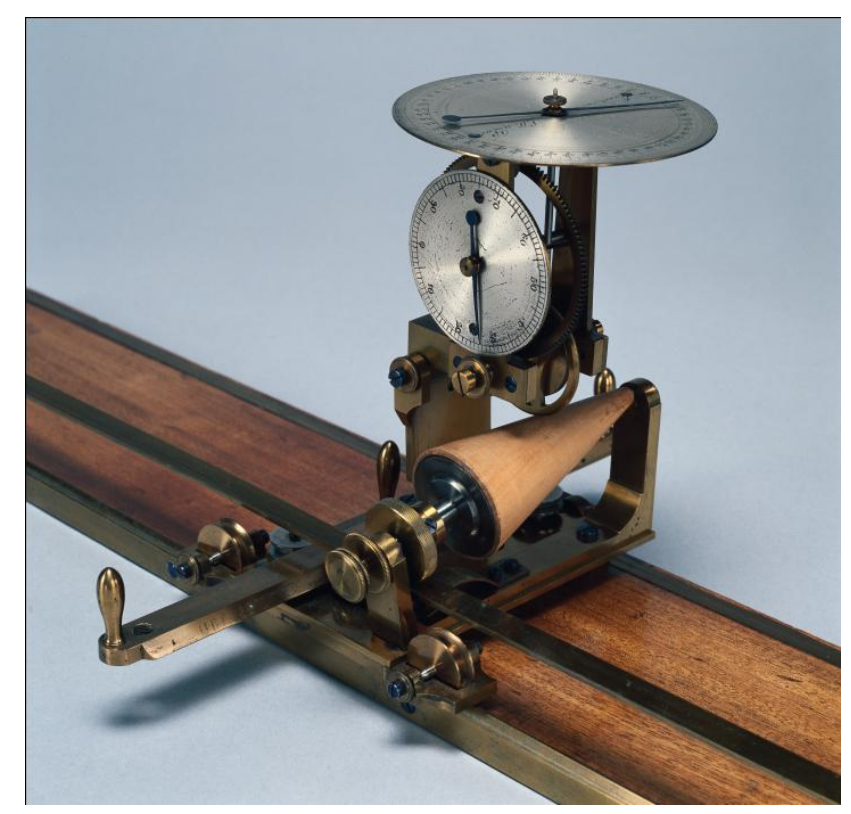

Fig. 1. Le planimètre à cône d'Oppikofer. Fabricant : Ernst, avant 1840.

Musée du CNAM, Inv. no. 02624-0000. Cliché : M. Favareille.

journee_mars08/journee08/durand-richard.html. Une exposition a d'abord nourri ce travail. Elle a eu lieu au Musée du CNAM de novembre 2006 à avril 2007, à partir d'un projet de recherche mené à REHSEIS sous la direction de Dominique Tournès. Le matériel de cette exposition, et de nombreux documents d'accompagnement, sont disponibles sur le site: "Les instruments du calcul savant »: http://www.reunion.iufm.fr/dep/mathematiques/calculsavant/index.html. 
Un opérateur déplace un pointeur le long du contour de la surface à mesurer. Le mécanisme de l'instrument décompose le mouvement du pointeur en un mouvement longitudinal et un mouvement latéral. Le mouvement longitudinal fait tourner le cône sur luimême autour de son axe. Le mouvement du cône entraîne celui de la roulette qui s'appuie sur une génératrice du cône, et le mouvement latéral déplace la roulette de telle sorte que sa distance au sommet du cône demeure égale au $\mathrm{y}=\mathrm{f}(\mathrm{x})$ de la courbe correspondant à ce tracé. Ainsi, à chaque déplacement infinitésimal du pointeur, la roulette tourne de $\mathrm{y} . \mathrm{dx}$ autour de son axe, dont la sommation donne l'intégrale cherchée. Le planimètre réalise donc mécaniquement l'intégration que le calcul est incapable de déterminer.

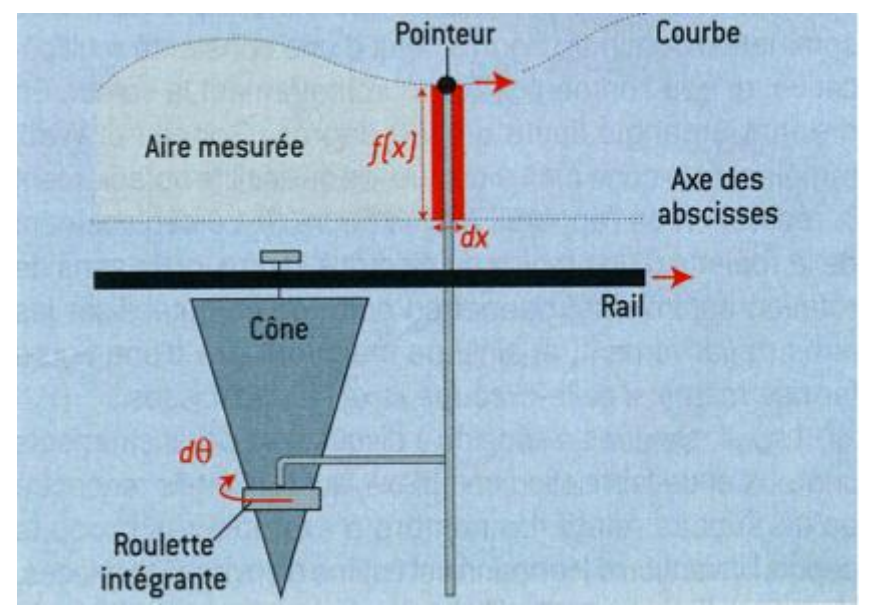

Fig. 2. Le principe du planimètre à cône. Schéma D. Tournès. Synopsis de l'exposition "Venez prendre l'aire! " (Cf. note 12)

Au milieu du siècle, la simplicité pratique du planimètre polaire, inventé et produit par Jakob Amsler (1823-1912) en Suisse, fonde définitivement le succès des planimètres. II réalise en coordonnées polaires ce que le planimètre à cône ou à disque - dits tous deux planimètres orthogonaux - réalisaient en coordonnées cartésiennes. Il est formé de deux bras articulés : à l'extrémité de l'un, le pôle reste fixe sur la carte ; à l'extrémité de l'autre, le pointeur suit le contour de la surface, et le mécanisme réalise l'intégration. Ses dimensions réduites permettent à l'ingénieur, l'architecte, l'arpenteur, de l'emporter partout, du chantier au bureau d'études. 


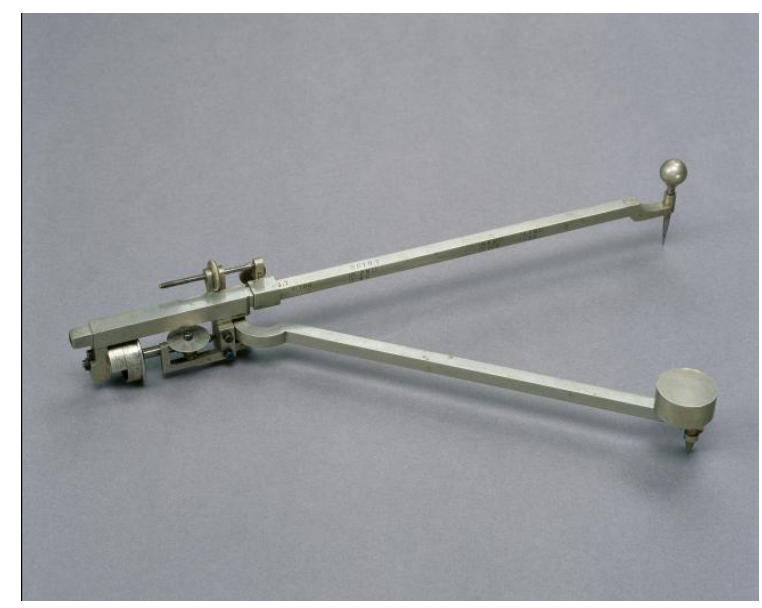

Fig. 3. Le planimètre polaire d'Amsler, concepteur et fabricant à Schaffhausen, 1865.

Musée du CNAM, Inv. 08004-0000. Cliché : M. Favareille.

Alors que le planimètre donne le résultat final de l'intégration, l'intégraphe trace la courbe intégrale au fur et à mesure du déplacement du pointeur. Lorsque le pointeur suit le contour de la courbe, le mouvement est transmis à une roulette coupante dont le plan reste parallèle à la tangente à la courbe. Ce faisant, cette roulette inscrit la courbe intégrale sur la feuille de papier enroulée sur un cylindre qui tourne sur lui-même en même temps qu'il se déplace longitudinalement.

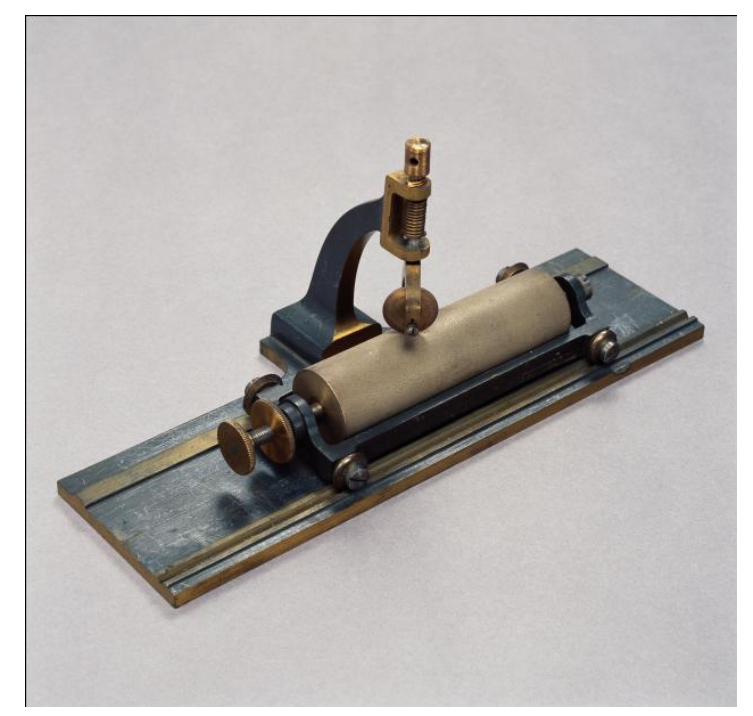

Fig. 4. Le principe de l'intégraphe : la vis à pas variable.

Misée du CNAM. Inv. 13300-004. Cliché : M. Favareille.

En France, plusieurs prototypes seront réalisés dans les années 1880 par l'ingénieur lituanien Bruno Abdank-Abakanowicz, par ailleurs chargé par le gouvernement français de l'électrification de la ville de Lyon. D'autres types d'intégraphes sont alors produits en Angleterre 
par des ingénieurs physiciens comme Charles Vernon Boys (1855-1944) et Henry Selby Hele Shaw (1854-1941), très soucieux en même temps des applications industrielles de ces dispositifs.

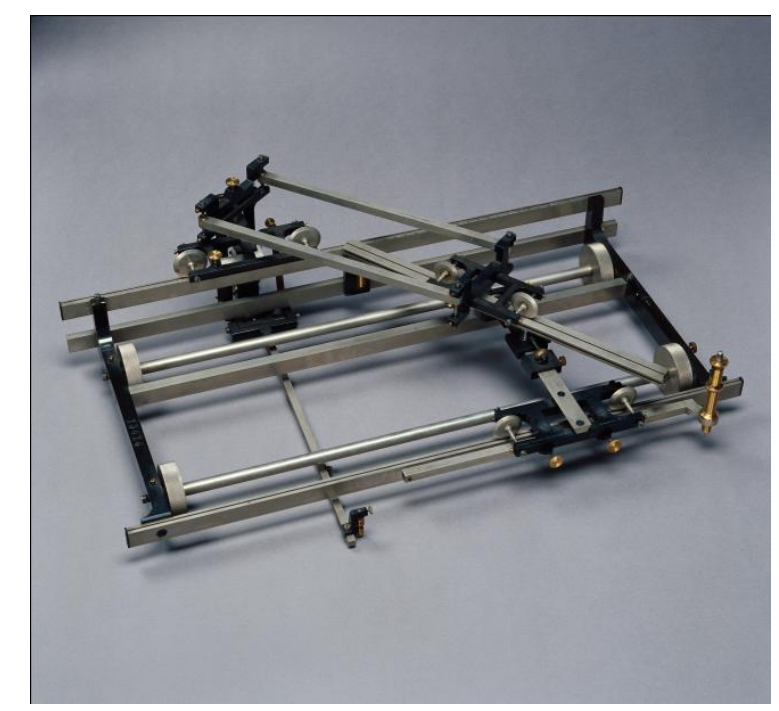

Fig. 5. Intégraphe avec transfert de direction par un parallélogramme articulé. AbdankAbakanowicz, Fabricant : Coradi, 1901. Musée du CNAM, Inv. 13242-0000. Cliché : M. Favareille.

Si la Grande-Bretagne ne semble pas particulièrement impliquée dans les prémices de ce développement, ses implications industrielles vont lui offrir de nouvelles potentialités. A la première Exposition Universelle de Londres en 1851, sont ainsi exposés deux planimètres à cône, celui de l'ingénieur écossais John Sang, et le grand planimètre - aujourd'hui perdu - propriété du Grand Duc de Toscane, fabriqué pour la mesure de l'île d'Elbe. Mais l'exposition de Crystal Palace marque surtout le triomphe de l'industrie britannique, du métier à tisser automatique à la machine à vapeur et aux navires métalliques, et le succès des planimètres des instruments de calcul d'aires n'y est alors pas tant celui des planimètres que celui des dynamomètres, destinés à mesurer le travail des machines pour en améliorer les performances. Ils ont été mis au point en France par Jean-Victor Poncelet (1788-1867) qui est aujourd'hui mieux connu comme fondateur de la géométrie projective que comme ingénieur savant, spécialiste des méthodes graphiques, et dont les cours de mécanique appliqués aux machines ont durablement marqué la science industrielle. Le dynamomètre a judicieusement incorporé le principe d'un planimètre où le cône est remplacé par un disque, puisque l'angle au sommet du cône ne joue aucun rôle dans la mesure à effectuer. Le cycle de fonctionnement étant enregistré graphiquement, l'aire de la 
surface définie par ce tracé donne le rendement de la machine. Le pays de la révolution industrielle offre à ces pratiques un remarquable champ d'investissement pour tous ces appareils.

\subsection{Prédiction des marées : de l'instrument à la machine de calcul}

Planimètres et intégraphes constituent donc le soubassement instrumental des machines qui vont être produites en Angleterre pour la prédiction des marées. Leur conception se nourrit cependant d'un contexte spécifique, celui des relations qui s'établissent entre science et industrie, notamment au sein de la British Association for the Advancement of Science. Délibérément créée en 1831 pour combler le fossé existant entre le monde industriel en mal de reconnaissance institutionnelle, et des institutions universitaires aussi traditionnelles que les universités anglicanes, elle joue délibérément ce rôle en organisant d'abord ses congrès alternativement entre villes industrielles et villes universitaires ${ }^{1}$. Mais surtout, elle structure de nombreux comités d'études coordonnant la réflexion de ces deux mondes autour de grands projets d'études dont l'impact économique et politique est de portée nationale, voire internationale. Un comité se réunit ainsi de 1867 à 1876 afin de « promouvoir le développement, l'amélioration et l'analyse harmonique des observations des marées ». Il est présidé par William Thomson, le futur Lord Kelvin (1824-1907). De 1867 à 1876, celui-ci réunit astronomes, mathématiciens, ingénieurs, calculateurs, amiraux et officiers de l'Amirauté. Ces relations nouvelles réalisent en quelque sorte le programme que prônait Babbage dans les années 1830, sans être pourtant directement impulsées par l'Etat comme il le recommandait. Aussi bien William Thomson que son frère, l'ingénieur James Thomson (1822-1892), en sont d'éclatantes manifestations. William, professeur de philosophie naturelle dans la grande ville industrielle de Glasgow de 1846 à 1904, est aussi directeur de l'Atlantic Telegraph \& Co depuis 1856. II conduit théoriquement et matériellement l'installation du premier câble transatlantique (1854-1866), avant de créer la firme Kelvin \& White, qui fabrique les nombreux instruments de précision qu'il met au point pour l'électricité et le télégraphe. Devenue Kelvin Bottomley \& Baird, cette firme fabriquera notamment des prédicteurs de marées jusqu'au milieu du $20^{e}$ siècle. William a reçu à Cambridge une solide formation mathématique, et étudie l'analyse de Fourier depuis les années 1840. Quant à son frère ingénieur, il a travaillé à Dublin, ainsi que dans les chantiers navals de Londres et de Manchester, avant de s'installer à Belfast, et d'y enseigner l'ingénierie à partir de 1854, puis d'occuper en 1873 la chaire d'ingénierie civile et de mécanique à l'université de Glasgow. James est l'inventeur de l'adaptation du principe du planimètre au système intégrateur disque-sphère-cylindre, mécanisme central de l'analyseur harmonique. Ce sont cette fois des

${ }^{1}$ Morrell, \& Thackray, Gentlemen of Science. 
surfaces polies qui roulent les unes sur les autres, évitant les problèmes de friction signalés par James Clerk Maxwell visitant Crystal Palace en 1851.
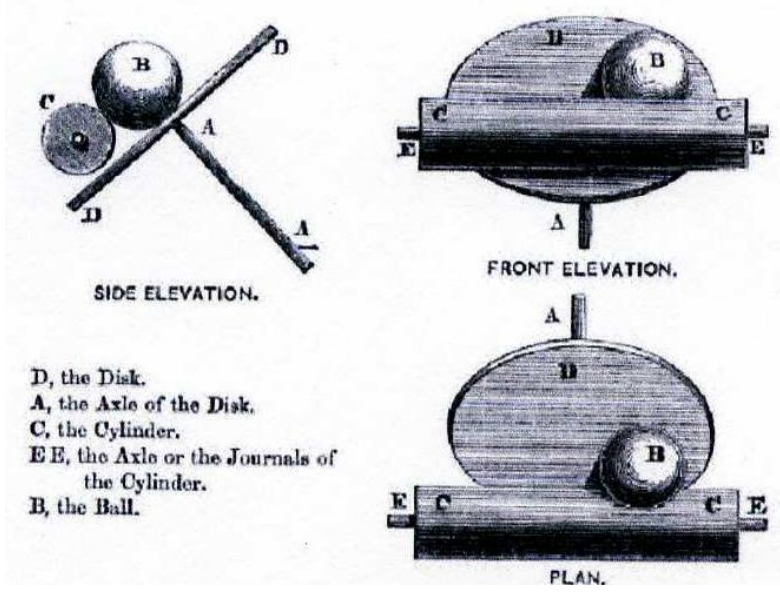

Fig. 6. Le système intégrateur disque-sphère-cylindre de James Thomson.

" An Integrating Machine Having a New Kinematic Principle », 1876, p. 492.

La prédiction des marées est en fait régie par la mise en œuvre successive de trois appareils. Depuis 1831, le marégraphe auto-enregistreur ${ }^{1}$ fournit l'enregistrement en continu de la variation de hauteur des marées dans un port donné en fonction du temps, un stylet dessinant une courbe sur un cylindre en rotation, à partir des mouvements d'un flotteur. Cette nouveauté automatise un programme systématique de mesures, assumée localement par les responsables portuaires. Une fois la courbe de marée obtenue pour un port quelconque, l'analyseur harmonique permet de décomposer la fonction périodique correspondant à la courbe enregistrée en autant d'harmoniques que de systèmes intégrateurs montés dans la machine, et d'obtenir ainsi les coefficients de Fourier de la décomposition. En effet, Joseph Fourier (1768-1830) a montré à propos de l'étude de la chaleur que toute fonction périodique $f(x)$ peut s'écrire sous la forme d'une série :

${ }^{1}$ Palmer, Henry, «Description of a Graphical Registrer of Tides and Winds ». Le marégraphe permet en outre de déterminer le niveau moyen de la mer à un endroit donné en éliminant les variations dues aux vagues et, sur un temps plus long, le niveau zéro (altitude) en éliminant les variations dues à la marée. Cette altitude zéro, qui correspond à la moyenne des basses mers de vive eau (grandes marées) peut faire office d'étalon et permettre d'établir les cartes marines et notamment les courbes de niveau.

${ }^{2}$ Antérieurement à cet appareil, les relevés visuels, soit sur une échelle de marée, soit sur un marégraphe non enregistreur, ne concernaient que les hauteurs de marée haute et marée basse. 
et que les coefficients de ce développement peuvent être calculés par intégration :

$$
A_{n}=\frac{1}{\pi} \int_{0}^{2 \pi} y \cos n \theta d \theta \quad B_{n}=\frac{1}{\pi} \int_{0}^{2 \pi} y \sin n \theta d \theta
$$

Le système disque-sphère-cylindre ${ }^{1}$ permet d'obtenir en général l'intégrale du produit de deux fonctions, et ici, dans le cas de l'analyseur harmonique, l'intégrale du produit d'une fonction par une fonction trigonométrique en sinus ou cosinus. La fonction y, lue par le pointeur sur la courbe enregistrée par le marégraphe et placée sur un cylindre en rotation, est transmise à chacun de ces systèmes. La distance du point de contact de la sphère au centre du disque est égale à y pendant la rotation du cylindre. Et la rotation d'un disque correspond à $\sin n$ [?d] ou cos $\mathrm{n}$ 国国. Chaque système intégrateur fournit un coefficient du développement en série.

Après un modèle expérimental réalisé en 1873, deux analyseurs harmoniques sont effectivement construits : I'un en 1876 avec 11 systèmes intégrateurs pour la prédiction des marées, l'autre en 1878 par la firme Munro, avec 7 composantes harmoniques pour le service météorologique. Ce dernier permet de soumettre à l'analyse harmonique les variations quotidiennes de la température, de la pression barométrique, et des composantes est-ouest de la vitesse du vent. II est également utilisé pour étudier l'influence lunaire sur les phénomènes météorologiques.

${ }^{1}$ Thomson, James, 1876, « An Integrating Machine Having a New Kinematic Principle ». 


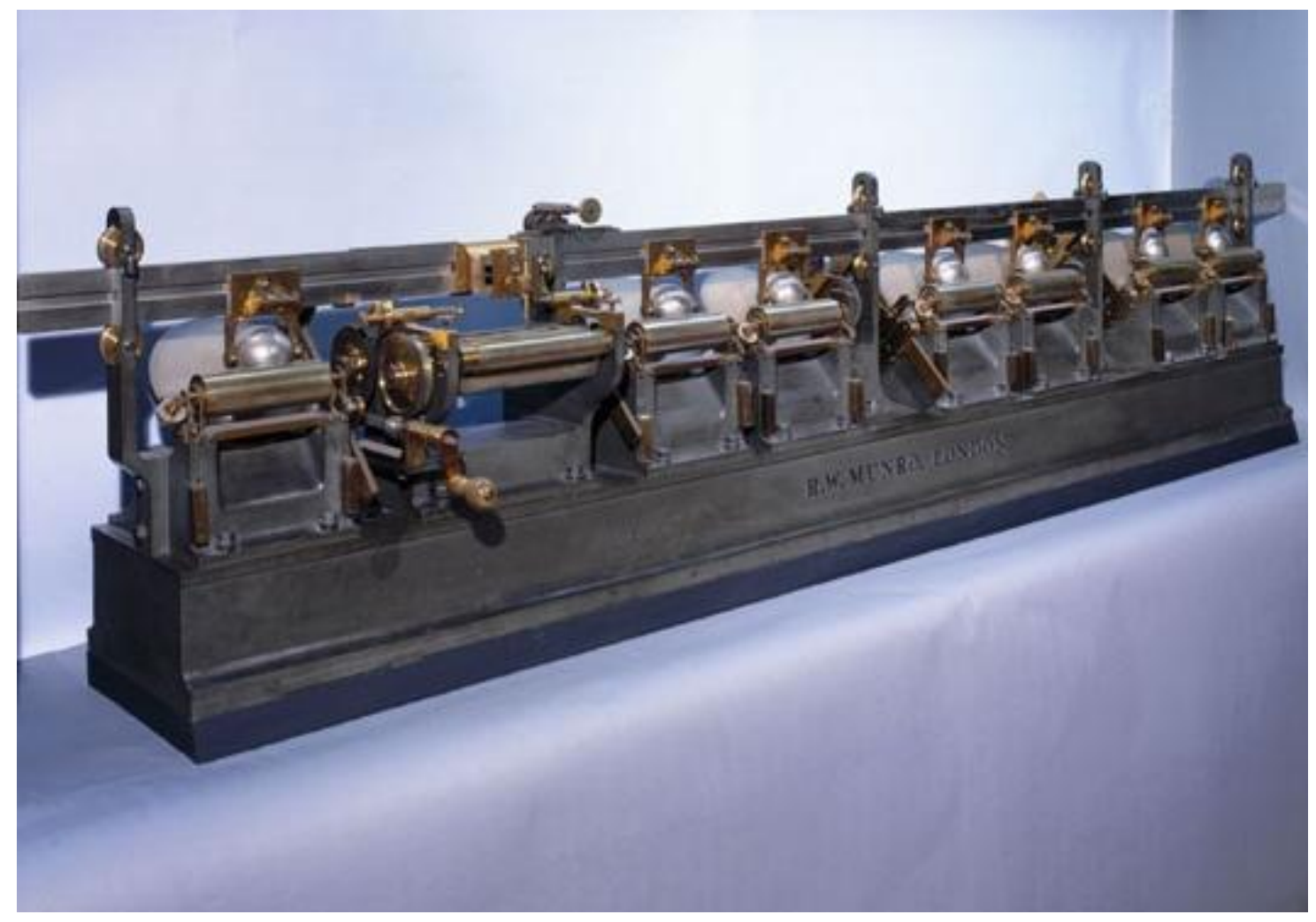

Fig. 7. Analyseur harmonique de Lord Kelvin. Fabricant : Munro, 1878.

Science Museum London SSPL. Inv. 1948-343

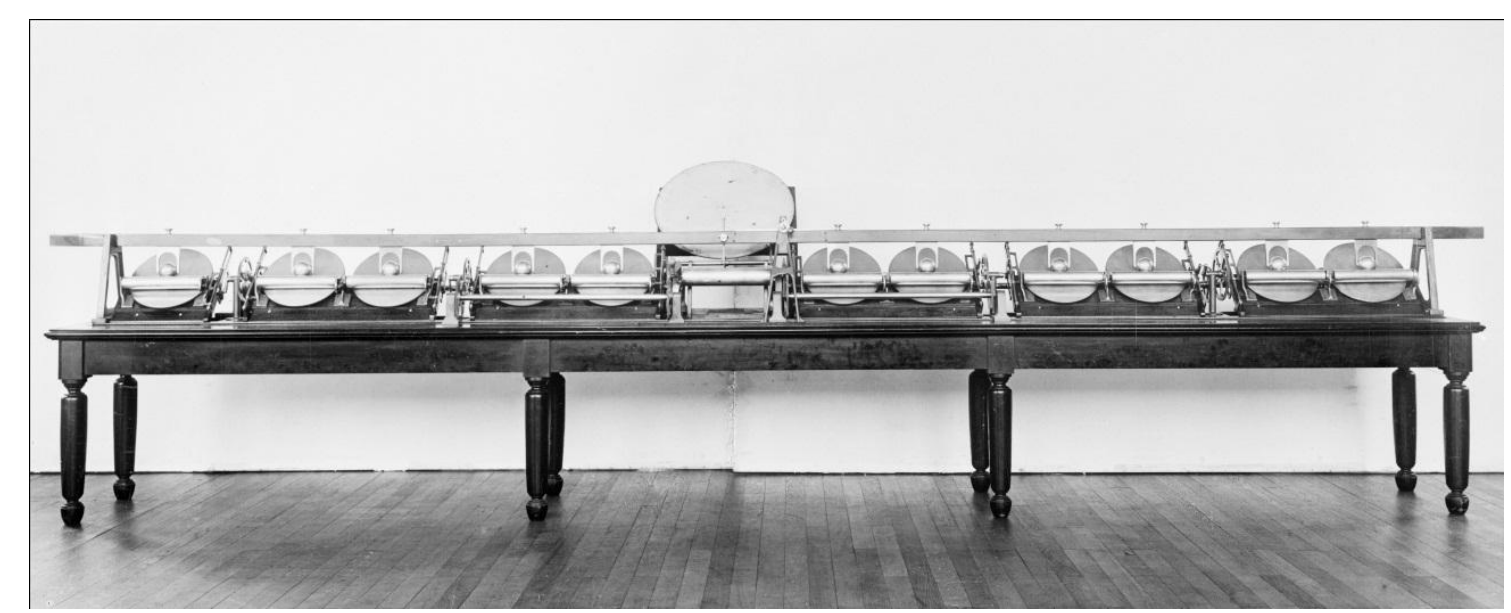

Fig. 8. Analyseur harmonique de Lord Kelvin., 1876.

Science Museum London SSPL. Inv. 1925-863. Blythe House.

Les coefficients des principales harmoniques des marées étant connues, celles-ci vont pouvoir être recomposées mécaniquement sur un troisième appareil, le Tide Predictor. Un fil 
flexible entoure alternativement une succession de poulies coplanaires, dont le mouvement harmonique vertical de chaque axe correspond à chacune des harmoniques à composer. L'une des extrémités du fil étant fixe, et l'autre étant munie d'un contrepoids qui le maintient tendu, le mouvement de cette seconde extrémité est deux fois la somme des mouvements des poulies. Un stylet dessine la trace de ce mouvement sur un papier graphique enroulé sur un cylindre en rotation $^{1}$.

Le prédicteur de marées peut ainsi effectuer en quatre heures une prévision des marées pour une année. La mise en œuvre de ces trois appareils sous-tend une étude expérimentale très approfondie de ces phénomènes, qui concerne en même temps la navigation et l'astronomie. En effet, les composantes de marée concernées ne sont pas seulement des multiples de la variable, comme peut le laisser supposer la présentation théorique ci-dessus de l'analyse de Fourier. Les harmoniques en question concernent les mouvements $d$ ' " astres fictifs " correspondants aux composantes semi-diurnes, diurnes, ter-diurnes, annuelles, etc., du soleil et de la lune. Laplace les a introduit comme équivalents heuristiques des termes du développement de la fonction potentiel de marée qu'il vient de définir. Elles engagent donc des connaissances en astronomie, en même temps qu'elles permettent d'en améliorer l'étude, comme celle de la théorie lunaire ${ }^{2}$.

Les rapports du comité de la British Association font état de l'importance de la collecte et du traitement des données d'observation, obtenus grâce à l'installation de marégraphes autoenregistreurs dans de très nombreux ports, bien au delà du Royaume-Uni : Karachi (Inde), Cat Island (Golfe du Mexique), San Diego et San Francisco (Californie), Fort Clinch (Port Ferdinand, Floride), Port Leopold et Beechey Island (Archipel Antarctique), mais aussi Toulon et Brest ${ }^{3}$. George H. Darwin (1845-1912) ${ }^{4}$, succédera à Kelvin à la présidence d'un nouveau comité de la British Association, afin de généraliser cette entreprise par la publication de rapports et d'ouvrages diffusant la méthode de réduction des marées par l'analyse harmonique. Son rapport ${ }^{5}$

${ }^{1}$ Lester, Description of the U.S. Coastand Geodetic Survey Tide-Predicting Machine $n^{\circ} 2$, Planche 1. Le modèle correspondant est exposé au Science Museum, Londres.

2 II faut rappeler en effet que le problème des trois corps n'a pas de solution analytique, et relève de méthodes d'approximation.

3 Thomson, William, «Report on a Committee appointed for the purpose of promoting the extension, improvement, and harmonic analysis of Tidal Observations ",. Semblables rapports sont déjà parus en 1868, 1869, 1870, 1871, 1872.

${ }^{4}$ George Darwin est le second fils de Charles Darwin, l'auteur de l'Origine des espèces publiée en 1859.

${ }^{5}$ Darwin, George, « Report on a Committee consisting of Professors G. H. Darwin and J.C. Adams, for the Harmonic Analysis of Tidal Observations ». 
de 1883 s'appuie sur l'existence d'un système organisé d'observations systématiques sur l'ensemble du territoire de I'Inde, où les Indian Tide Tables sont publiées sous l'autorité du Secrétariat d'Etat.

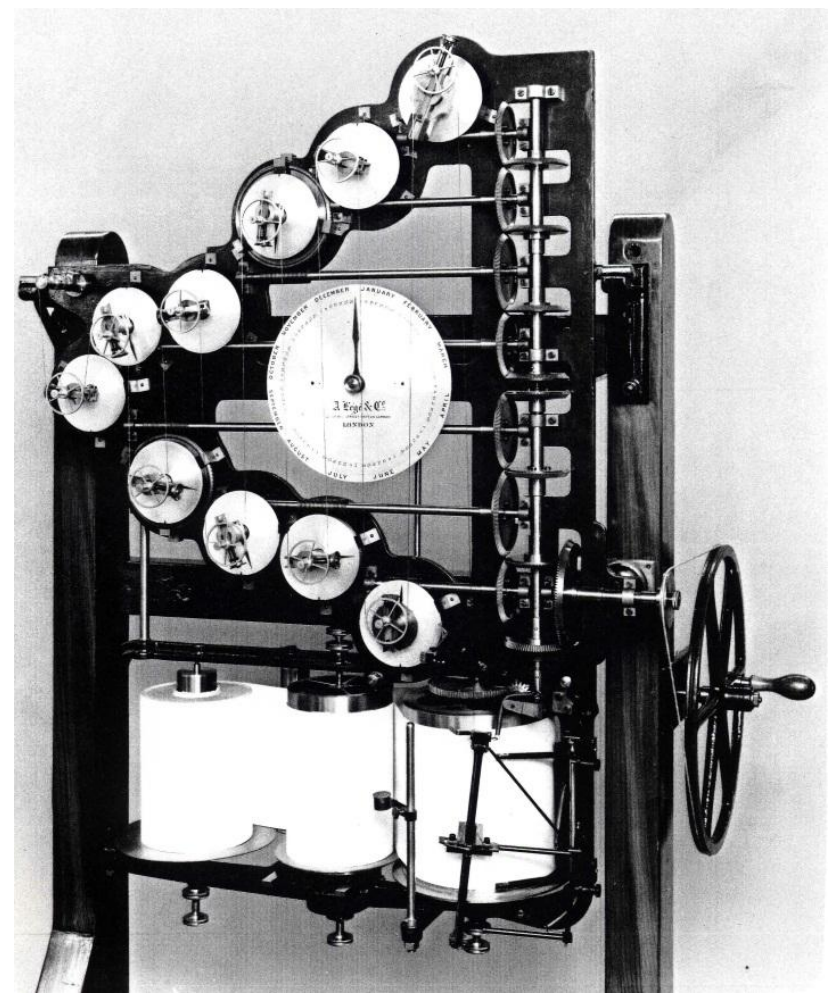

Fig. 9. Le prédicteur de marées $n^{\circ}$ 2. Science Museum London. Inv. 1876-829.

Description et cliché E. Lester, planche 1.

\subsection{Ce qui fait sens dans la prédiction des marées depuis le 19e siècle}

Cet investissement sur la prédiction des marées est éminemment signifiante pour l'empire britannique tel qu'il se constitue au $19^{\mathrm{e}}$ siècle. Du point de vue scientifique, il marque le couronnement d'une période où se modifient les relations entre théorie et pratique autour de cette question. Au moment de la création de la British Association, en 1831, les mathématiciens britanniques ont dû s'initier rapidement au formalisme analytique mieux développé sur le Continent, afin d'assimiler la Mécanique Céleste et le Système du Monde de Pierre Simon de Laplace (1749-1827) ${ }^{1}$. La théorie laplacienne marque une avancée certaine sur la compréhension

${ }^{1}$ Durand-Richard, «L'Ecole Algébrique Anglaise : les conditions conceptuelles et institutionnelles 
du phénomène des marées, puisqu'elle inclut la réponse dynamique des océans à la théorie newtonienne de l'attraction exercée par le Soleil et la Lune, débouchant sur l'analyse harmonique et la prédiction des marées. Elle fait néanmoins encore l'hypothèse simplificatrice d'un océan $d^{\prime}$ 'épaisseur constante ${ }^{1}$ couvrant toute la planète Terre. Elle laisse ainsi percevoir que la structure ondulatoire du phénomène des marées est beaucoup plus complexe que ce qui avait été envisagé jusqu'alors. Dans le climat de compétition économique et politique avec la France, il est alors urgent, y compris pour les savants britanniques, une fois surmonté leur handicap théorique quant aux méthodes analytiques, de dépasser les limitations de la théorie laplacienne pour un meilleur contrôle des océans. La compétition entre la France et l'Angleterre est alors aussi bien économique et politique que scientifique. Le développement de l'empire britannique engage une maîtrise des océans et des ports pour pénétrer des contrées et des ports lointains, où le régime des marées diffère considérablement du rythme semi-diurne - environ deux marées hautes et deus marées basses par vint quatre heures - couramment observé sur la côte Est de l'Atlantique, en raison des phénomènes astronomiques et de la géographie maritime et côtière locale. Ce basculement de l'observation des mouvements de la mer le long des côtes à une compréhension globale du mouvement des mers, essentielle à cette nation maritime, associe les besoins de l'astronomie et de la navigation dans un même effort concerté. II s'appuie sur un programme général d'observations des variations de hauteur des marées, et de raffinement de la théorie laplacienne, qui se poursuit de 1831 aux années 1870 et au delà, comme en témoignent deux grandes séries d'articles de William Whewell ${ }^{2}$ (1794-1866) et John Lubbock (1803-1865), à partir de données fournies essentiellement par l'amiral Sir Francis Beaufort (1774-1857). Ce programme vise notamment à établir des cartes cotidales joignant par les lignes de niveau les points des océans où la pleine mer a lieu en même temps, étude que la théorie ondulatoire de Kelvin permet effectivement de raffiner quarante ans plus tard.

L'énorme quantité de données à prendre en compte et de calculs à effectuer pour résoudre les équations différentielles introduites par Laplace pour la prédiction des marées ${ }^{3} n^{\prime} e s t$ pas la seule raison qui conduit au projet des frères Thomson. Aussi bien en France qu'en

d'un calcul symbolique comme fondement de la connaissance ».

${ }^{1}$ ou variant linéairement en fonction de la co-latitude du lieu.

${ }^{2}$ Leurs articles sont publiés des années 1830 aux années 1850 et portent sur les observations des marées, d'abord dans les ports de Londres, Liverpool, Plymouth et Singapour. Ils sont publiés à la fois dans les publiés dans les Philosophical Transactions of the Royal Society, et sous forme de rapports pour la British Association.

${ }^{3}$ Même avec l'introduction des ordinateurs dans les centres marégraphiques, il faudra attendre quelques décennies avant que les ordinateurs soient assez puissants pour représenter en détail l'océan dans sa globalité. 
Angleterre, les observations sont suffisamment régulières, et portent sur un intervalle de temps suffisamment long pour que les irrégularités puissent être réduites par le calcul. En effet, aussi bien la méthode de Lubbock en Angleterre que celle de Chazallon en France, bien adaptées aux marées semi-diurnes présentant une inégalité diurne faible, permettent de prédire les marées en ces lieux par des méthodes d'approximation, et en effectuant les calculs à la main. Mais la situation diffère pour les ports éloignés ${ }^{1}$ de l'empire britannique, comme ceux de l'Inde et de l'Australie, où les marées ont de fortes inégalités diurnes, et qui seront les premiers à bénéficier de l'utilisation de ces machines.

Après le premier prédicteur de marées construit par la firme A. Légé \& Co en 1876, composant dix harmoniques, le second, produit pour le Indian Office, est conçu par Edward Roberts (1845-1933), secrétaire et calculateur du comité présidé par Kelvin. Composant 24 harmoniques, il sera utilisé de 1879 à 1903 pour dresser les tables de marées des principaux ports de I'Inde. Roberts réalisera ensuite en 1906 un prédicteur pour 40 harmoniques, qu'il exploitera lui-même à des fins commerciales. Parallèlement, aux Etats-Unis, le US Coast and Geodetic Survey, sous l'impulsion de William Ferrel (1817-1891), suit avec un énorme intérêt le développement des prédicteurs de marées en Angleterre. II réalise nouveau prédicteur de marée en 1882, avec 19 composantes, qui sera utilisé intensivement jusqu'en 1912. II sera remplacé en 1912 par une machine à 37 composantes, qui fonctionnera jusqu'à l'introduction des ordinateurs en 1966. Le Service Hydrographique français fera l'acquisition de deux de ces prédicteurs de marées : I'un en 1901, construit en 1880 par la firme Kelvin \& White ${ }^{2}$ dans les années 1880, avec 17 composantes, permettant d'établir les tables de marées pour 32 ports de l'empire colonial français, l'autre ${ }^{3}$ en 1950, construit par la firme Kelvin, Bottomley \& Baird Ltd, avec 30 composantes, qui fonctionnera jusqu'en 1966. Cette même firme vend alors différents modèles de prédicteurs : au Brésil en 1910, au Japon en 1914 et en 1924, à l'Argentine en 1918, au Canada en 1927. L'Allemagne n'est pas en reste, avec ses deux imposants prédicteurs, réalisés chacun au début des deux guerres mondiales par la firme Otto Töpfer \& Sohn.

${ }^{1}$ Cartwright, Tides, A Scientific History.

${ }^{2}$ Il est aujourd'hui au Musée du Conservatoire National des Arts et Métiers, mais non exposé.

${ }^{3}$ Voir Fig. 10. Il se trouve actuellement dans le hall du Service Hydrographique et Océanographique Marine à Brest. 


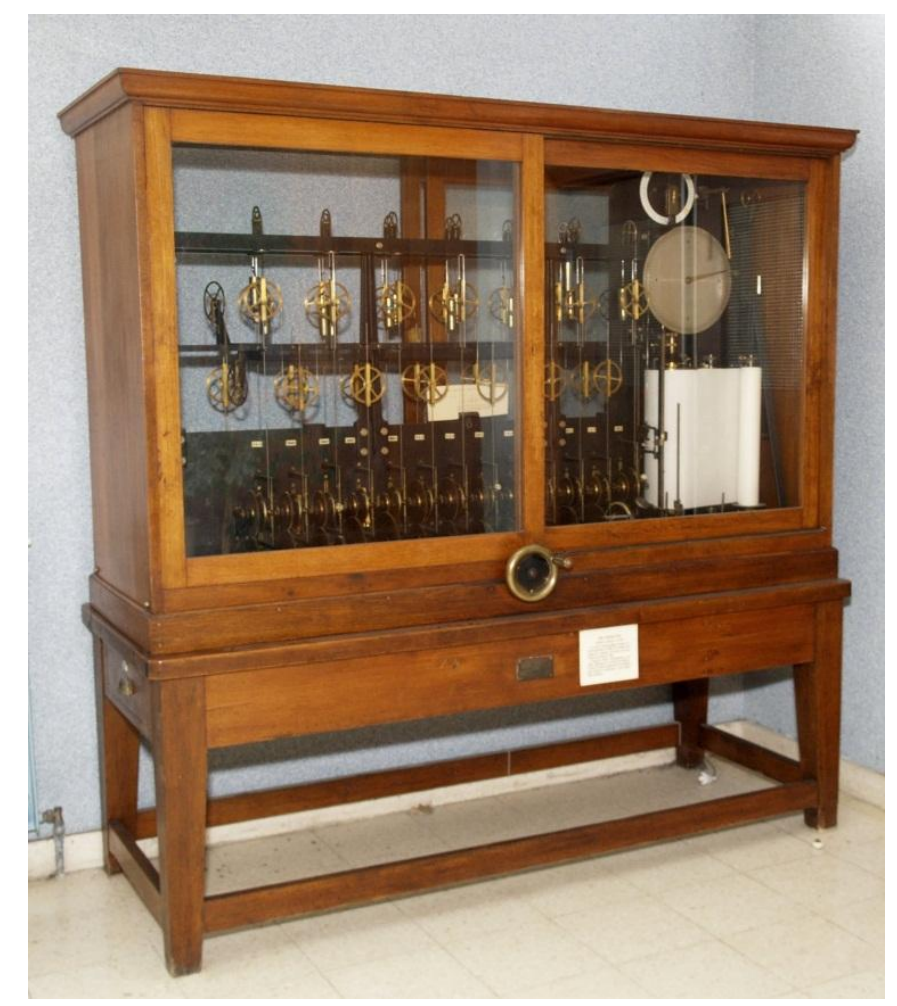

Fig. 10. Prédicteur de marées $n^{\circ}$ 6. Constructeur : Kelvin, Bottomley \& Baird.

Acquis par le SHOM en 1950. En fonction jusqu'en 1966. Localisation : SHOM, Brest.

Cliché : Frédérique Plantevin (Université de Brest).

Deux firmes britanniques, la firme Chadburn et la firme Kelvin, Bottomley \& Baird Ltd exportent ainsi ces machines dans le monde entier, dans tous les pays qui n'ont pas de fabrication propre, jusqu'en Norvège ou en Russie. Elles travaillent sous le contrôle du Liverpool Tidal Institute, créé en 1919 par le mathématicien Joseph Proudman (1888-1975), toujours sous l'impulsion de la British Association. Son successeur Arthur T. Doodson (1890-1969) est dans les années 1950 l'autorité mondiale en matière de prédiction des marées.

\section{Conclusion}

Ingénieurs, mathématiciens, physiciens et astronomes ont ainsi joué un rôle essentiel au XIXème siècle pour concevoir les moyens mécaniques de dépasser les limites théoriques du calcul infinitésimal. La situation sociétale de leur travail commun, à la rencontre du politique, du technique et du scientifique, est significative des enjeux sous-jacents à la mise en acte des mathématiques dans la société. La prédiction des marées en est un cas particulier. Elle met en avant l'importance de ces relations dans l'empire britannique, où la question de ces relations a longuement et explicitement été débattue dans les cercles scientifiques depuis les ruptures d'équilibre introduites par la Révolution Industrielle. Si la navigation est aujourd'hui relativement oubliée depuis le développement de l'aviation, elle est stratégiquement cruciale à cette époque, 
d'une part, dans une perspective de maîtrise politico-économique du monde opposant la GrandeBretagne et la France par le biais de leurs empires coloniaux, et d'autre part, en établissant des perspectives communes aux affaires contingentes et aux vérités nécessaires régissant l'équilibre du système solaire.

Ce contexte permet de percevoir que la signification des mathématiques n'est pas seulement logique. Les mathématiques sont également signifiantes de par la représentation du monde qu'elle permet de soutenir dans la société, longtemps assimilée tantôt à une pure lecture de vérités cachées, tantôt à la notion de progrès. Dans le cadre du thème de réflexion auxquelles ces journées sont consacrées, donner voie à ces implications - qui ne sont pas que des applications - des mathématiques permettrait aux élèves et aux étudiants de percevoir les différents facteurs qui les rendent signifiantes, du " pur » - ou plutôt du « simple »- jeu de l'esprit aux fonctions sociales qui les concernent en tant que citoyens en devenir.

\section{Bibliographie}

Abdank-Abakabovicz, Bruno, Les intégraphes, Paris, Gauthier-Villars, 1991.

Austin, John L., Quand dire, c'est faire, Paris, Seuil, 1970.

Babbage, Charles, 1820, The Philosophy of Analysis, manuscrit inédit, British Library, Add. Mss 37202, pp. 42-43.

Boole, George, Les lois de la pensée, traduction de An Investigation of the Laws of Thought, on which are founded the mathematical Theories of Logic and Probabilities, par Souleymane Bachir Diagne, Paris, Vrin, 1992 (1854).

Cartwright, David Edgar, Tides, A Scientific History, Cambridge, Cambridge University Press, 1999.

Chemla, Karine, et Shuchun Guo Les neuf chapitres: le classique mathématique de la Chine ancienne et ses commentaires, traduction française avec des addenda détaillés et une édition commentée du texte chinois du livre et de son commentaire, Paris, Dunod, 2004.

Darwin, George, « Report on a Committee consisting of Professors G. H. Darwin and J.C. Adams, for the Harmonic Analysis of Tidal Observations ", Report of the fifty-first Meeting of the British Association for the Advancement of Science, held in Southport, 1883, pp. 49-118.

Descartes, René, La Géométrie, in (éds) Adam, Ch., \& Tannery, P., CEuvres, Paris, 1902 (1637), tome VI, p. 6 .

Durand-Richard, Marie-José, 1996, "L'Ecole Algébrique Anglaise : les conditions conceptuelles et institutionnelles d'un calcul symbolique comme fondement de la connaissance ", in (éd.) C. 
Goldstein, J. Gray, J. Ritter, L'Europe mathématique - Mythes, histoires, identités, Paris, Editions de la Maison des sciences de I'homme, pp. 445-498.

Lester, E., Description of the U.S. Coastand Geodetic Survey Tide-Predicting Machine $n^{\circ} 2$, Washington, Goverment Printing Office, Department of Commerce, U.S. Coast and Geodetic Survey, 1915.

Locke, John, Essai philosophique sur l'entendement humain, , traduction de M. Coste, Paris, Vrin, $1983(1700)$.

Morrell, Jack, \& Thackray, Arnold, Gentlemen of Science, Early Years of the British Association for the Advancement of Science, Clarendon Press, Oxford, 1981.

Palmer, Henry, "Description of a Graphical Registrer of Tides and Winds", Philosophical Transactions of the Royal Society, 1831 vol. 121, pp. 209-214.

Ramunni, Girolamo, "L'ordinateur entre continuité et rupture », in (dir.) Durand-Richard, MarieJosé, Les mathématiques dans la cité, Paris, Presses universitaires de Vincennes, 2006.

Ricœur, Paul, De l'interprétation, Paris, Seuil, 1965.

- La métaphore vive, Paris, Seuil, 1975.

--Du texte à l'action, essais d'herméneutique, Paris, Seuil, 1986.

Thomson, James, 1876, "An Integrating Machine Having a New Kinematic Principle », Proceedings of the Royal Society, 1876, $n^{\circ} 24$, pp. 262-65. Republié dans Thomson, William \& Tait, Peter Guthrie, Treatise on Natural Philosophy, vol. 1, Partie 1, Cambridge, Cambridge University Press, $2^{\text {ème }}$ édtn, 1879, pp. 488-492.

Thomson, William, «Report on a Committee appointed for the purpose of promoting the extension, improvement, and harmonic analysis of Tidal Observations ", Report of the forty-fifth Meeting of the British Association for the Advancement of Science, held in Glasgow, 1876, pp. 275307.

Tournès Dominique, "Les instruments du calcul savant ", 2007, http://www.reunion.iufm.fr/dep/mathematiques/calculsavant/index.html.

Volkov, Alexeï, "Raisonnement par analogie dans les mathématiques chinoises ", in DurandRichard, Marie-José (dir.), L'analogie dans la démarche scientifique, perspective historique, Paris, L'Harmattan, 2008.

Marie-José Durand-Richard. Univ Paris Diderot, Sorbonne Paris Cité, SPHERE, UMR 7219, CNRS, F75205 Paris, France. mjdurand.richard@gmail.com 